The Medical Training Initiative scheme in forensic psychiatry: reflections on training and practice in the UK and Nigeria

\author{
Nosa Igbinomwanhia, ${ }^{1} \odot$ Boladale Mapayi, ${ }^{2}$ James Lee $^{3}$ and \\ Derek K. Tracy ${ }^{4}$
}

${ }^{1} \mathrm{MBBCH}$, FWACP, MRCPsych, Specialty Registrar in Psychiatry, South London and Maudsley NHS Foundation Trust, Lewisham Coundation Trust, Lewsham Community Mental Health for mentia London, UK. Email: nosa.igbinomwanhia@nhs.net

${ }^{2} \mathrm{MBChB}$, FWACP, MClinPsychol, $\mathrm{PhD}$, Consultant Psychiatrist, Obafemi Awolowo University Teaching Hospitals Complex, Ile-Ife, Nigeria

${ }^{3}$ MRCPsych, Consultant Forensic Psychiatrist, Memorial Hospital, Oxleas NHS Foundation Trust, London, UK

${ }^{4}$ FRCPsych, Consultant Psychiatrist and Medical Directo West London NHS Trust, London,

Keywords. Transcultural psychiatry; education and training; forensic mental health services; RCPsych MTI; international medical graduates.

First received 25 Apr 2021 Final revision 10 Aug 2021 Accepted 7 Dec 2021

doi:10.1192/bji.2021.63

(c) The Author(s), 2022. Published by Cambridge University Press on behalf of the Royal College of Psychiatrists. This is an Open Access article, distributed under Access article, distributed und Creative Commons Attribution licence (https://creativecommons.org/ licenses/by/4.0/), which permits unrestricted re-use, distribution, and reproduction in any medium, provided the original work is properly cited.
The Royal College of Psychiatrists' Medical Training Initiative (MTI) enables qualified foreign-trained psychiatrists to gain further experience within the UK National Health Service (NHS). This article summarises the observations of two Nigerian psychiatrists during their forensic psychiatry placements on the MTI scheme and two of their UK supervisors. It discusses similarities and differences between Nigeria and the UK in forensic psychiatry, including the use of mental health legislation, the scope of forensic psychiatry and elements of the pathway to qualification in both countries. It concludes with recommendations that could improve the robustness of the MTI scheme.

The Royal College of Psychiatrists (RCPsych) Medical Training Initiative (MTI) scheme enables international psychiatry graduates to train and practise in the UK's National Health Service (NHS), acquiring skills and experience for up to 2 years before returning to their home countries. ${ }^{1}$

Authors B.M. and N.I. had completed their undergraduate and postgraduate medical training in Nigeria and subsequently worked at consultant level before joining the scheme in 2018, rotating across forensic psychiatry settings, including low secure, medium secure and a category B prison, the Criminal Justice Liaison Team (court diversion) and community forensic mental health teams. J.L. and D.K.T. were two of their MTI supervisors. The authors herein reflect on this interesting career experience and its learning points, compare aspects of forensic psychiatric practice in the UK and Nigeria, and highlight future opportunities of the MTI scheme.

\section{The scope of forensic psychiatry in} Nigeria and the UK (England and Wales) Forensic psychiatry deals with the interface between psychiatry and the law, and with the flow of mentally disordered offenders along a continuum of social systems. ${ }^{2}$ Like other branches of healthcare, it is a reflection of the social system within which its services are delivered; cultural differences in the UK and Nigeria make it unsurprising that there are significant differences in the nature and extent of forensic psychiatry service delivery between these countries. Further, Nigeria has 4.1 physicians per 10000 population, compared with 28.1 per 10000 in the UK (2007-2013), and about 300 psychiatrists for a country with a population of about 200 million. Forensic psychiatry therefore revolves mostly around managing mentally disordered offenders within prisons or in general psychiatric hospitals, assessing offenders at the request of the police, or giving expert opinions to courts, either in oral form or as written reports at the instruction of the court or a solicitor.

Ogunlesi \& Ogunwale ${ }^{3}$ gave a three-model description of forensic psychiatry practice in Nigeria in the context of custodial facilities. First-line mental healthcare is commonly provided by non-psychiatric doctors, nurses and allied staff in prison clinics. A second element is care provided by visiting psychiatrists (and multidisciplinary teams where available) from a regional tertiary health facility. They also described a third model similar to the second but in which nursing care is provided by a stationed mental health nurse from the same tertiary health facility. In all three models medication is funded by the prison service or directly by the inmate's relatives. However, in some parts of Nigeria, there exist alternative informal arrangements whereby prison medical staff, who may or may not have specialist mental health training and are usually nurses, identify inmates who are in need of psychiatric care and refer these to the psychiatric hospital or visiting psychiatric teams for evaluation and treatment; psychiatrists visit weekly or fortnightly. Nigerian law provides for the transfer of severely ill mentally disordered offenders to named psychiatric hospitals for assessment and treatment, ${ }^{3,4}$ but a lack of adequately secured facilities in such hospitals is a major limitation to the implementation of this legislation.

Elements of forensic psychiatry outside custodial care are mostly practised in unstructured interface with courts, police services and the prisons. There are usually no dedicated multidisciplinary forensic teams, perhaps a reflection of staff shortage across the spectrum of mental health professionals in the country. 
It is important to note that dedicated criminal justice liaison teams (police stations and court diversion) that are often the first point of contact with mental health services for mentally disordered offenders in the UK do not currently exist in Nigeria. Other key elements of the framework for delivery of forensic psychiatry services in the UK, including graded secure units, forensic community teams and supported accommodation, are not present, although historically there were semblances of secure units in the form of prison-based asylums. ${ }^{3}$

\section{Training in forensic psychiatry in Nigeria and the UK (England and Wales)}

In the UK core trainees seek placements for higher training to qualify with a certificate of completion of training (CCT). An alternative to this is the certificate of eligibility for specialist registration (CESR) route. ${ }^{5}$

Higher forensic training in the UK involves mainly treating offenders who have committed crimes when mentally ill or who become unwell in prison, with placements in various settings, including prison, the community and medium and high secure hospitals. The 3-year programme is overseen by the RCPsych and local training programme directors, with each trainee having an educational supervisor to oversee and monitor the trainee's progress. A placement clinical supervisor and the trainee meet weekly to ensure the completion of competencies set out in the RCPsych curriculum ${ }^{6}$ and Good Medical Practice guidelines and to conduct measured workplacebased assessments. This aspect of weekly supervisory meetings between supervisor and trainee was a major learning point for the MTI trainees.

There is a particular focus on developing skills in structured actuarial risk assessment, formulation of offending in the mentally disordered offender population and, of course, the law related to forensic psychiatry. Skills related to becoming an expert witness are developed by the management of current cases proceeding through the courts. Higher training also focuses on the key leadership competencies required for consultant practice and includes the requirement to achieve competencies in clinical governance, quality, and service design and development. The trainee is expected also to seize opportunities for teaching and research at each stage of the training.

The pathway to forensic psychiatry practice in Nigeria is less clearly developed. Doctors would usually enter residency training in psychiatry in one of the government-owned teaching hospitals or stand-alone psychiatric hospitals. This training is generally for a period of 6 years, during which the doctor undertakes rotations in general adult psychiatry and other psychiatry subspecialties, such as child and adolescent (including intellectual disability through the life cycle), consultation liaison psychiatry, neurology, old age psychiatry, community, social and rehabilitation psychiatry, addiction psychiatry, psychotherapy, emergency psychiatry and forensic psychiatry. These rotations are at junior trainee level for 3 years of 6-monthly rotations, at the end of which the doctor sits the Membership examination of either or both of two Colleges: the National Postgraduate Medical College of Nigeria (NPMCN) and the West African College of Physicians (WACP). This examination usually takes the form of written essays, objective structured clinical examinations (OSCEs), picture tests and clinical clerkship of patients. Successful candidates in this examination proceed to senior trainee level, known as senior registrars, for another minimum of 3 years, again rotating through subspecialties at 6-monthly intervals.

Unlike UK psychiatry (but similar to UK surgical specialties), at the end of 3 years of senior trainee rotations, candidates are expected to sit the Fellowship examinations of either or both Colleges. The examination at this stage takes the form of a dissertation paper written under the supervision of a senior consultant; the dissertation goes through an assessor, who is usually another senior consultant psychiatrist, and is subsequently 'defended' in the final examination, when the candidate is also expected to pass OSCE-type questions. Candidates must be successful in their dissertation paper and in the OSCE to be awarded the Fellowship of either College; this qualifies the candidate to be appointed to a consultant post in Nigeria, or in the case of the West African Fellowship, anywhere in the West African subregion.

The formative and summative assessments for training appear quite robust, although training in the forensic psychiatry subspecialty in Nigeria for now remains largely informal and without a specific qualification pathway. ${ }^{7,8}$ It largely consists of selfmotivated attendance at relevant local and international courses, conferences and seminars, and obtaining a diploma/degree in related academic disciplines.

However, the two major training colleges in the country have recently taken giant strides in reviewing existing curricula to standardise training in the various subspecialties, including forensic psychiatry. The proposed curriculum is quite specific in terms of competencies and duration of training for various subspecialties in psychiatry.

\section{The watchful eyes of the Mental Health Act (MHA)}

'What section is the patient on?', 'When is their section due for renewal?', 'When is their next tribunal date?' are examples of familiar questions in UK forensic psychiatry practice that would hardly ever pop up in forensic psychiatry practice in Nigeria at the current time. Nigeria's current mental health legislation is derived from the colonial Lunacy Ordinance, which was enacted as the Lunacy Ordinance in 1916, last revised as the Lunacy Act in $1958 .^{9}$ It does not take patient consent into account and offers little opportunity to individuals to contest their detention. The Act is not clear on plans regarding treatment, uses what are now derogatory terms for the mentally ill and makes no provision for the rehabilitation and resettlement 
of mentally disordered offenders. ${ }^{10}$ In particular, there is no section specifically tailored to the needs of the mentally disordered offender (to compare with, for example, sections 37 and 37/41 of the UK's Mental Health Act 1983 (MHA)) and it is therefore perhaps unsurprising that, in our experiences, the Act is barely referred to in routine forensic psychiatry practice in Nigeria. ${ }^{9}$ This would be a disorienting experience for a UK-trained forensic psychiatrist, who has to continually practise within the ever-watchful ambit of the MHA 1983, revised in 2007.

Throughout our (N.I. and B.M.) MTI placement, our practice was consciously framed by an active and relevant MHA that felt almost labyrinthine in its use. It was a new experience having to work with consultants, psychiatric trainees and other professionals on multidisciplinary teams who frequently made recourse to the MHA in patient care. To the MTI trainee from Nigeria new to a UK forensic placement, these MHA processes can easily appear unduly bureaucratic and laborious, with much form-filling and report writing and numerous tribunal and court attendances, and would seem to impinge on the time for direct clinical care of the patient.

Notable aspects of UK mental healthcare is that almost all patients detained under the MHA are legally eligible for section 117 aftercare in the community and for the care programme approach (CPA) with 6-monthly multidisciplinary team meetings with the patient to discuss all aspects of their care and treatment. Mentally disordered offenders, therefore, whether in hospital or in the community, are supported by the mental health service.

As MTI trainees, we quickly learned that much of our patient care was going to revolve around MHA paperwork under consultant supervision. It is noteworthy that tribunal sittings and managers' hearings, CPA-like structures and section 117 meetings are currently not reflected in forensic psychiatry practice in Nigeria.

\section{Constraints to the MTI trainee on forensic placements}

There are a few challenges that Nigerian trained psychiatrists on the MTI scheme are likely to face. The structure of service delivery differs significantly and it is important for UK supervisors to recognise this in their interactions with their new MTI trainees. Thankfully, the RCPsych has provided guidance for NHS trusts interested in employing MTI doctors to vacant posts and it strongly recommends that this guidance is read by employing bodies. ${ }^{1}$ The guidance elaborately describes the nature of the MTI post, highlighting its focus on training and encouraging trusts to provide necessary support in this regard. It also describes the RCPsych's expectations of trusts and seeks to identify what support is available to help the relocating doctor settle into their new role and adjust to life in the UK. The guidance encourages robust interaction between the MTI doctor and the trust throughout the application process and also highlights pastoral areas that trusts could possibly focus on in making the MTI doctor's relocation as seamless as possible. It is important for supervising consultants to read this guidance as they are key to the scheme meeting the expectations of the MTI trainee and those of the RCPsych. Notably, the RCPsych has made additional efforts at providing employing bodies with information pertaining to MTI posts, including informative and best practice workshops and we encourage employing trusts to take advantage of such opportunities.

The need to prioritise familiarisation with the workings of the UK MHA, rigorous identification protocols for prison placements and sometimes unclear delineation of the status of the MTI trainee require working through. The struggle to acclimatise while at the same time needing to be a functional part of a multidisciplinary team, meeting family needs in a new environment and building new social networks are other challenges an MTI trainee would need to surmount. ${ }^{11,12}$ The RCPsych's guidance to trusts ${ }^{1}$ and the Academy of Medical Royal Colleges' relocation guide ${ }^{12}$ provide useful information to both trusts and the relocating MTI doctor and we recommend that these documents are read by both parties.

\section{Concluding thoughts}

The RCPsych MTI scheme remains a viable route for international medical graduates (IMGs) to acquire much needed knowledge and skills in the UK while also contributing their knowledge and skills to the UK healthcare delivery system, but it would benefit from more formal guidance.

MTI trainees who attempt and pass the MRCPsych examinations during the period of their placement should be given the opportunity to have their MTI placement scaled up to Specialty Trainee Year 4 (ST4) level; this would further enhance their ability to acquire needed competencies to take back to their home countries, which is a core objective of the scheme.

Exposure to UK forensic psychiatry practice provided an opportunity to reflect on and contrast the current status of forensic training in Nigeria. Although the subspecialty training is still in early stages, progress is being made to strengthen the curriculum and improve competence for the forensic psychiatrist. In terms of specific modifications to Nigerian mental health legislation to make it more accepting of international best practice, a lot of advocacy has gone into driving the necessary engine to accept the latest revision of the Mental Health Bill, pioneered by the Association of Psychiatrists in Nigeria. When signed into law, this new legislation will cover most of the areas of deficiency and will improve the practice of forensic psychiatry in Nigeria. While it continues to be useful for trainees from Nigeria to utilise the MTI scheme to improve their understanding of psychiatry practice outside Nigeria, it would also be beneficial for the RCPsych to organise reverse 
programmes where trainees from the UK come to Nigeria to experience psychiatric practice. It would facilitate the exchange of ideas and they may pick up ingenious ways to solve complex problems in the absence of robust mental health legislation. This would need consideration by local UK trusts and deaneries and Health Education England to assure appropriateness of placement, training specifications and overall goal of the exercise. It might also be useful for senior UK psychiatrists, especially those who supervise MTI trainees, to spend some time in Nigeria. They would learn about the complexities of practice and might even begin to understand what the country's training encompasses to enable progress in placements, so that Nigerian MTI trainees are not allocated to posts that are way below their level of expertise.

\section{Data availability}

Data availability is not applicable to this article as no new data were created or analysed in this study.

\section{Author contributions}

N.I. and B.M. contributed Nigerian perspectives on Forensic Psychiatry training and practice and MTI trainee experience. J.L. and D.T. contributed perspectives on UK Forensic psychiatry training and practice.

\section{Funding}

This research received no specific grant from any funding agency, commercial or not-for-profit sectors.

\section{Declaration of interest None.}

\section{References}

1 Royal College of Psychiatrists. Guidance for Trusts Employing an RCPsych MTI International Fellow. RCPsych, 2019 (https://www. rcpsych.ac.uk/docs/default-source/training/medical-training-initiative/ rcpsych-mti-trust-guidance-2019.pdf).

2 Arboleda-Florez J. Forensic psychiatry: contemporary scope, challenges and controversies. World Psychiatry 2006; 5: 87-91.

3 Ogunlesi AO, Ogunwale A. Correctional psychiatry in Nigeria: dynamics of mental healthcare in the most restrictive alternative. BJPsych Int 2018; 15: 35-8.

4 Federal Ministry of Justice. Criminal Procedure Act [Nigeria], Cap C41 Vol. 4. Federal Ministry of Justice, 2004.

5 Royal College of Psychiatrists. Routes to registration. RCPsych, 2021 (https://www.rcpsych.ac.uk/training/your-training/routes-toregistration)

6 Royal College of Psychiatrists. A Competency Based Curriculum for Specialist Training in Psychiatry: Specialists in General Psychiatry. RCPsych, 2019 (https://www.rcpsych.ac.uk/docs/default-source/ training/curricula-and-guidance/general_psychiatry_curriculum_march_ 2019.pdf).

7 Ogunlesi AO, Ogunwale A, De Wet P, Roos L, Kaliski S. Forensic psychiatry in Africa: prospects and challenges. Afr J Psychiatry 2012; 15: 3-7.

8 Abdalla-Filho E, Bertolote JM. Forensic psychiatric systems in the world. Rev Bras Psiquiatr 2006; 28: 56-61.

9 Igbinomwanhia NG, Akanni O. Constraints, ethical dilemmas and precautions in psychiatric practice within non-contemporaneous mental health laws: a Nigerian experience with involuntary commitment. J Law Policy Glob 2019; 87: 21-5.

10 Westbrook $\mathrm{AH}$. Mental health legislation and involuntary commitment in Nigeria: a call for reform. Wash U Global Law Rev 2011; 10: 397-418.

11 Lagunes-Cordoba E, Maitra R, Dave S, Matheiken S, Oyebode F, O'Hara J, et al International medical graduates: how can UK psychiatry do better? BJPsych Bull 2021; 45: 299-304.

12 Academy of Medical Royal Colleges. Medical Training Initiative: Relocation Guide. Academy of Medical Royal Colleges, 2018 (https://www.aomrc.org.uk/wp-content/uploads/2018/05/MTI Relocation_Guide_MAY2018-v3-wr-1.pdf).

\title{
THEMATIC PAPER \\ Perinatal mental health around the world: priorities for research and service development in The Netherlands
}

\author{
Iris Leppers, ${ }^{1} \odot$ Cornelis Pieter Matthijs Veth, ${ }^{2}$ Dieuwertje Anna de Waardt, ${ }^{2}$ \\ Hanneke Migchels ${ }^{3}$ and Maria Johanna Traa ${ }^{4}$
}

${ }^{1} \mathrm{MD}$, Psychiatry Resident, Department of Psychiatry, Elisabeth-Tweesteden Hospital, Tilburg, The Netherlands. Email i.leppers@etz.n

${ }^{2} \mathrm{MD}$, Specialist Psychiatrist, Department of Psychiatry,

Elisabeth-Tweesteden Hospital, Tilburg, The Netherlands

${ }^{3} \mathrm{MD}$, Specialist Obstetrician, Department of Obstetrics and Gynaecology, ElisabethTweesteden Hospital, Tilburg, The
The Netherlands has an unique system of perinatal and postpartum healthcare. Pregnancy care is delivered predominantly by primary care midwives and childbirth services predominantly involve hospital care. The first week postpartum, all women receive daily care from maternity nurses. In addition, hospitals and out-patient clinics offer perinatal mental healthcare. More specifically, 'POP care' (psychiatry, obstetrics and paediatrics) was developed to promote multidisciplinary collaboration in this regard. Although clinical practices and government initiatives to improve pregnancy-related mental healthcare work well, they have yet to be fully described and evaluated. The current COVID-19 pandemic has an impact on health services and perinatal mental health. 